\title{
LETTER
}

Chronic myelogenous leukemia

\section{Primary therapy and relative survival among elderly patients with chronic myeloid leukemia: a population-based study in the Netherlands, 1989-2017}

\author{
Geneviève I. C. G. Ector $\mathbb{D}^{1,2} \cdot$ Otto Visser $^{3} \cdot$ Peter E. Westerweel ${ }^{4} \cdot$ Jeroen J. W. M. Janssen $^{5} \cdot$ Nicole M. A. Blijlevens ${ }^{1}$ • \\ Avinash G. Dinmohamed $\mathbb{1}^{5,6,7,8}$
}

Received: 25 March 2020 / Revised: 25 May 2020 / Accepted: 29 May 2020

(c) The Author(s) 2020. This article is published with open access

\section{To the Editor:}

The advent of the first tyrosine kinase inhibitor (TKI) imatinib in 2001-targeting the BCR-ABL1 oncoproteinrevolutionized the management of chronic myeloid leukemia (CML). Trials of imatinib and subsequent TKIs in CML have shown excellent survival outcomes. However, the broad applicability of trial results in daily practice has its limits because of the patient selection criteria dictated by study protocols—especially among elderly, often comorbid

Supplementary information The online version of this article (https:// doi.org/10.1038/s41375-020-0902-1) contains supplementary material, which is available to authorized users.

Geneviève I. C. G. Ector

genevieve.ector@radboudumc.nl

1 Department of Hematology, Radboud University Medical Center, Nijmegen, The Netherlands

2 Department of Hematology, Radboud IKNL, Department Research and Development, Nijmegen, The Netherlands

3 Department of Registration, Netherlands Comprehensive Cancer Organisation (IKNL), Utrecht, The Netherlands

4 Department of Internal Medicine, Albert Schweitzer Hospital, Dordrecht, The Netherlands

5 Department of Hematology, Cancer Center Amsterdam, Amsterdam University medical centers, location VUmc, Amsterdam, The Netherlands

6 Department of Research and Development, Netherlands Comprehensive Cancer Organisation (IKNL), Utrecht, The Netherlands

7 Department of Hematology, Cancer Center Amsterdam, Amsterdam University Medical Centers, Location AMC, Amsterdam, The Netherlands

8 Department of Public Health, Erasmus University Medical Center, Rotterdam, The Netherlands patients who are generally underrepresented in pivotal CML trials [1].

Population-based studies can inform how changing treatment practices affect population-level survival. The paucity of representative population-based studies in CML is comparatively outdated and does not explicitly provide a comprehensive apprehension on treatment or survival among different older age groups, as patients are frequently combined into one age group or broad ranges of age [2-5]. Therefore, to complement and extend on prior populationbased research, we conducted a comprehensive, nationwide, population-based study to assess trends in primary treatment and survival among elderly CML patients diagnosed during a 28-year period in the Netherlands.

We selected all CML patients aged $\geq 70$ years diagnosed between 1989 and 2017-with survival follow-up through January 1, 2019-from the nationwide population-based Netherlands Cancer Registry (NCR) using International Classification of Diseases for Oncology morphology codes 9863 and 9875 . Nationwide since 1989, the NCR has an overall coverage of $>95 \%$ of all newly diagnosed malignancies in the Netherlands [6]. The NCR relies on case notification through the Nationwide Network of Histopathology and Cytopathology, and the Nationwide Registry of Hospital Discharges (i.e., inpatient and outpatient discharges). Information on the dates of birth and diagnosis, sex, prior malignancies, vital statistics (i.e., alive, emigration, or death), and first-line anti-CML treatment-defined as chemotherapy, immunotherapy, or TKI-was available for individual patients. This information is collected by trained registrars of the NCR through retrospective medical records review. Data on the use of TKI therapy and the specific therapeutic agent were registered in the NCR for patients diagnosed from 2007 and 2014, respectively. Of note, the use of TKI therapy before the calendar year 2007 was recorded in the NCR as anti-CML treatment. 
Fig. 1 Primary treatment of elderly patients with chronic myeloid leukemia in the Netherlands. a Shows information on primary therapy according to age at diagnosis and calendar period of diagnosis among patients diagnosed between 1989 and 2017. b Shows information on primary therapy according to age at diagnosis among patients diagnosed between 2014 and 2017. The table in (a) presents the proportion of patients receiving a particular treatment within a specific age group and calendar period. The absolute number of patients within a specific age group and calendar period presented in (a) is shown in Supplementary Table 3. The table in (b) presents the absolute number and proportion of patients receiving a particular treatment within a specific age group.
A -Anti-CML therapy $\approx$ TKI $\square$ CT alone : Other/unknown therapy $\square$ No therapy

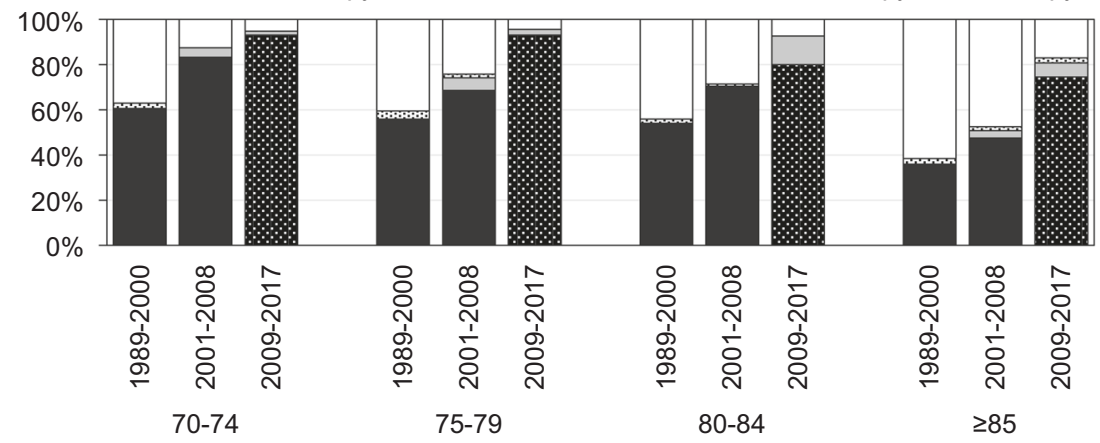

Age at diagnosis and calendar period of diagnosis

\begin{tabular}{lcccccccccccc}
\hline Primary therapy & \multicolumn{10}{c}{ Column percentage } \\
\hline Anti-CML therapy & 61 & 83 & - & 56 & 69 & - & 54 & 70 & - & 36 & 47 & - \\
TKI & - & - & 93 & - & - & 93 & - & - & 80 & - & - & 74 \\
CT alone & - & 4 & 2 & - & 6 & 3 & - & - & 13 & - & 3 & 6 \\
Other/unknown therapy & 2 & - & - & 4 & 1 & - & 2 & 1 & - & 3 & 2 & 2 \\
No therapy & 37 & 13 & 5 & 40 & 24 & 4 & 44 & 29 & 7 & 62 & 47 & 17 \\
\hline
\end{tabular}

B

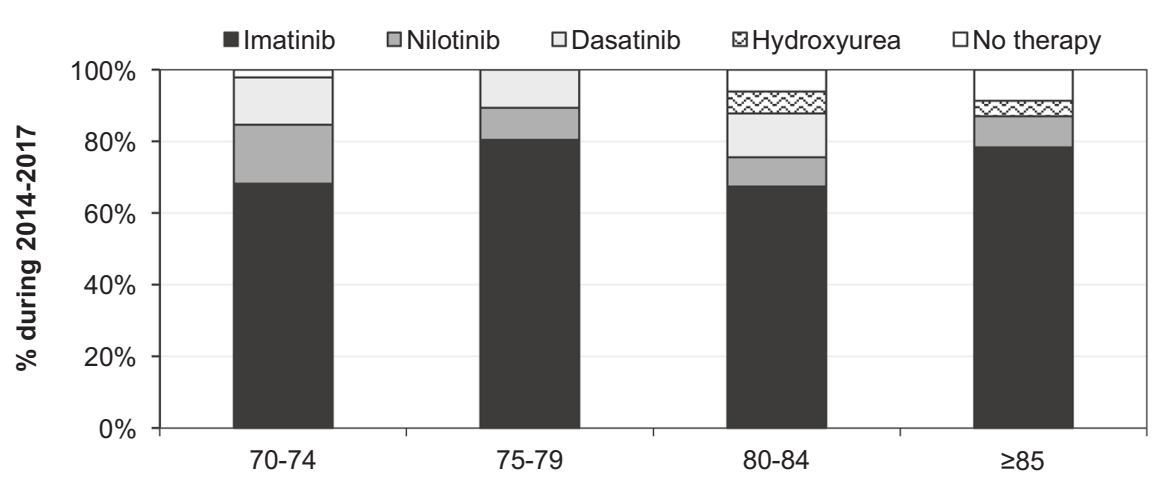

Age at diagnosis

\begin{tabular}{lccccccccccc}
\hline Primary therapy & \multicolumn{2}{c}{ Imatinib } & \multicolumn{2}{c}{ Nilotinib } & \multicolumn{2}{c}{ Dasatinib } & \multicolumn{2}{c}{ Hydroxyurea } & \multicolumn{2}{c}{ No therapy } & Total \\
\hline Age, years & $\mathbf{N}$ & $\mathbf{( \% )}$ & $\mathbf{N}$ & $\mathbf{( \% )}$ & $\mathbf{N}$ & $\mathbf{( \% )}$ & $\mathbf{N}$ & $\mathbf{( \% )}$ & $\mathbf{N}$ & $\mathbf{( \% )}$ & $\mathbf{N}$ \\
\hline $70-74$ & 62 & $(68)$ & 15 & $(16)$ & 12 & $(13)$ & 0 & - & 2 & $(2)$ & 91 \\
$75-79$ & 45 & $(80)$ & 5 & $(9)$ & 6 & $(11)$ & 0 & - & 0 & - & 56 \\
$80-84$ & 33 & $(67)$ & 4 & $(8)$ & 6 & $(12)$ & 3 & $(6)$ & 3 & $(6)$ & 49 \\
$\geq 85$ & 18 & $(78)$ & 2 & $(9)$ & 0 & - & 1 & $(4)$ & 2 & $(9)$ & 23 \\
\hline Total & $\mathbf{1 5 8}$ & $\mathbf{( 7 2 )}$ & $\mathbf{2 6}$ & $\mathbf{( 1 2 )}$ & $\mathbf{2 4}$ & $\mathbf{( 1 1 )}$ & $\mathbf{4}$ & $\mathbf{( 2 )}$ & $\mathbf{7}$ & $\mathbf{( 3 )}$ & $\mathbf{2 1 9}$ \\
\hline
\end{tabular}

Patients were divided into four age groups (70-74, $74-79,80-84$, and $\geq 85$ years) and three calendar periods (1989-2000, 2001-2008, and 2009-2017). The first calendar period represents the pre-TKI era, the second and third period the era in which imatinib and subsequent generations of TKIs were introduced in daily practice, respectively.

Relative survival (RS) was calculated to estimate disease-specific survival as per the cohort methodology. RS was calculated up to 5 years after diagnosis according to the calendar period of diagnosis and age at diagnosis and measured from diagnosis to death, emigration, or end of follow-up (January 1, 2019), whichever occurred first. RS is the overall survival in the patient cohort divided by the expected survival of a comparable group from the general population, matched to the patients through age, sex, and period [7]. The expected survival was calculated from Dutch population life tables-stratified by age, sex, and period-according to the Ederer II methodology [8]. Poisson regression was applied to investigate linear trends in RS over time and the relative excess risk of mortality [9]. A $P<$ 0.05 indicates statistical significance. The Privacy Review Board of the NCR approved the use of anonymous data for this study. 
Fig. 2 Relative survival of elderly patients with chronic myeloid leukemia in the Netherlands according to age at diagnosis and calendar period of diagnosis, 1989-2017. Relative survival rates (RSR) are shown for the following age categories: a 70-74 years, b 75-79 years, c 80-84 years, and $\mathbf{d} \geq 85$ years. The table presents the projected 1-, 3-, and 5-year RSR with 95\% confidence intervals (CIs) according to age at diagnosis and calendar period of diagnosis. $* P$ value for likelihood ratio test assessing linear trends from the period 1989-2000 to the period 2009-2017.

$$
=1989-2000
$$

70-74 years

A

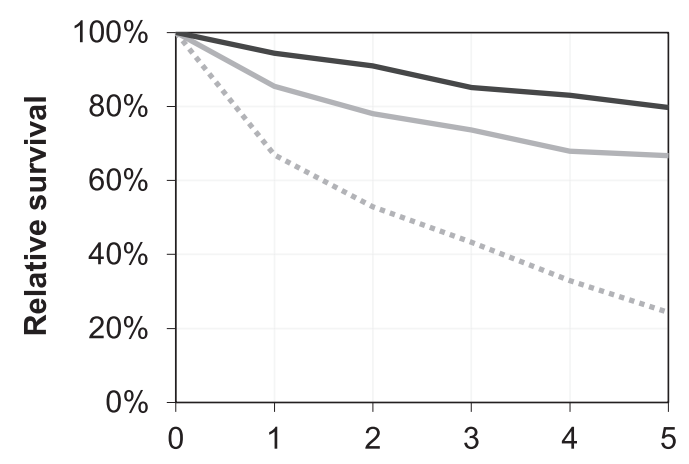

2001-2008

B

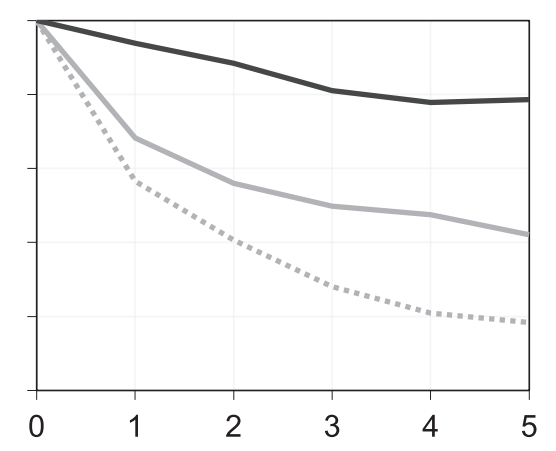

Years from diagnosis

\begin{tabular}{lllll}
\hline \multicolumn{4}{c}{ Calender period } \\
\hline RSR & $1989-2000$ & $2001-2008$ & $2009-2017$ & \multirow{2}{*}{$\begin{array}{c}P \text { for } \\
\text { trend }\end{array}$} \\
\cline { 2 - 4 } & \multicolumn{3}{c}{ RSR (in\%) with 95\% Cl } & $<0.001$ \\
1-year & $67(60-73)$ & $85(76-92)$ & $94(89-98)$ & $<0$ \\
3-year & $43(36-51)$ & $74(62-83)$ & $85(78-91)$ & $<0.001$ \\
5-year & $24(18-31)$ & $67(54-77)$ & $80(70-88)$ & $<0.001$ \\
\hline
\end{tabular}

\begin{tabular}{|c|c|c|c|}
\hline \multicolumn{3}{|c|}{ Calender period } & \multirow{3}{*}{$\begin{array}{l}P \text { for } \\
\text { trend }\end{array}$} \\
\hline $1989-2000$ & $2001-2008$ & $2009-2017$ & \\
\hline \multicolumn{3}{|c|}{ RSR (in $\%$ ) with $95 \% \mathrm{Cl}$} & \\
\hline $57(49-63)$ & $68(59-76)$ & $94(87-98)$ & $<0.001$ \\
\hline $28(22-35)$ & $50(40-59)$ & $81(70-90)$ & $<0.001$ \\
\hline $18(13-25)$ & $42(32-52)$ & $79(65-90)$ & $<0.001$ \\
\hline
\end{tabular}

C

80-84 years

D $\geq 85$ years
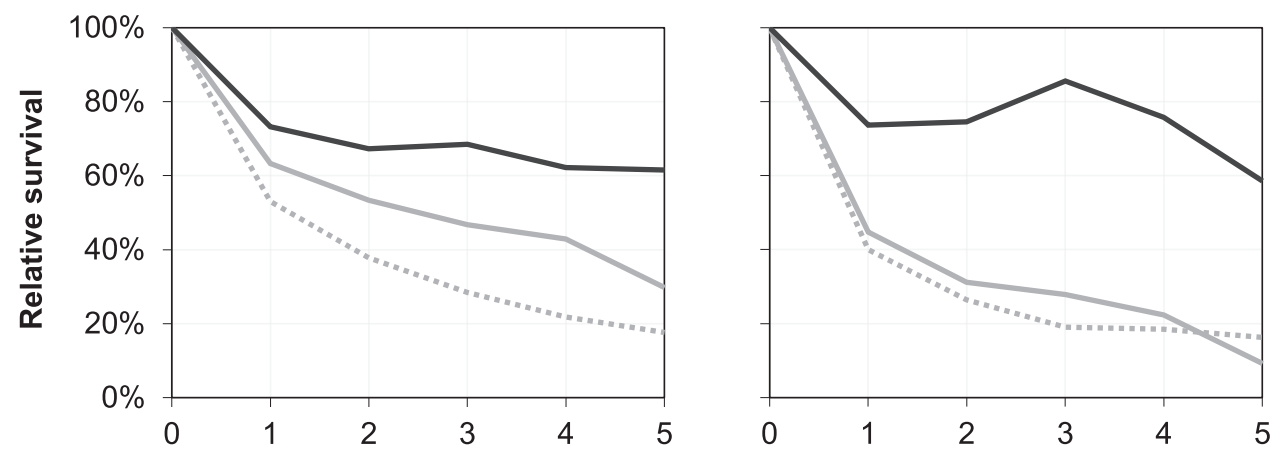

Years from diagnosis

\begin{tabular}{lllll}
\hline \multicolumn{4}{c}{ Calender period } \\
\cline { 1 - 3 } RSR & $1989-2000$ & $2001-2008$ & $2009-2017$ & \multirow{2}{*}{$\begin{array}{c}P \text { for } \\
\text { trend }\end{array}$} \\
\cline { 2 - 4 } 1-year & $53(44-62)$ & $63(52-73)$ & $73(62-82)$ & 0.007 \\
3-year & $28(20-38)$ & $47(35-59)$ & $69(55-80)$ & $<0.001$ \\
5-year & $18(10-27)$ & $30(19-43)$ & $62(45-78)$ & $<0.001$ \\
\hline
\end{tabular}

A total of $1525 \mathrm{CML}$ patients aged $\geq 70$ years were included in the study (median age, 77 years; range 70-95 years; $56 \%$ males; and $11 \%$ with a prior malignancy). The characteristics of these patients are presented in Supplementary Table 1 . Overall, $31 \%, 32 \%, 22 \%$, and $15 \%$ of patients were aged $70-74,75-79,80-84$, and $\geq 85$ years, respectively.

Figure 1a presents information on primary treatment according to age at diagnosis and calendar period of diagnosis. The application of anti-CML therapy increased over time across all age groups. Data of patients diagnosed during 2009-2017 showed that 93\%, 93\%, 80\%, and 74\% of the patients across the four age groups received a TKI, respectively. Detailed data on primary therapy among 219 patients diagnosed during 2014-2017 are shown in Fig. 1b.

Figure 2 shows RS according to age at diagnosis and calendar period of diagnosis. One-, 3-, and 5-year RS improved significantly over time across all age groups. 
Nevertheless, patients across all age groups experienced ongoing excess mortality - as compared with the general population-in the most recent period (2009-2017). Furthermore, improvement in RS among patients aged $\geq 75$ lagged behind those aged 70-74 years in the period between 1989-2000 and 2001-2008. The most pronounced improvement in RS among patients aged $\geq 75$ years took place between 2001-2008 and 2009-2017, especially among patients aged $\geq 85$ years.

Next, we analyzed the relative excess risk of mortality within five years after CML diagnosis in a multivariable model (Supplementary Table 2). Overall, when simultaneously adjusted for sex, age at diagnosis, calendar period of diagnosis, and a prior malignancy, patients diagnosed in 2009-2017 had 63\% lower excess mortality, as compared with patients diagnosed in 2001-2008 (excess mortality ratio, $0.37 ; 95 \%$ confidence interval $[\mathrm{CI}] ; 0.29-0.50 ; P<$ 0.001 ). However, after additional adjustment for primary therapy, the effect of calendar period lost statistical significance. This finding suggests that changes in the application of primary therapy contributed to the improved survival during 2009-2017. Lastly, older age and male sex were independent predictors of poor prognosis.

In this nationwide, population-based study, we demonstrated that RS among all age groups of CML patients aged $\geq 70$ years improved considerably after the introduction of imatinib in 2001. However, the improvement between 1989-2000 and 2001-2008 among patients aged $\geq$ 75 years was less pronounced compared with patients aged $70-74$ years. The improvement in the former age group was most pronounced between 2001-2008 and 2009-2017, especially among patients aged $\geq 85$ years. This observation might suggest that patients aged $\geq 75$ years did not immediately gain benefit from the advances in CML management.

Our findings that survival improvements lag in the oldest age groups are mostly congruent with and extend on findings from the few available population-based studies in CML [2, 3, 10, 11]. In the USA and England, 5-year RSRs (95\% CI) among patients aged 65-74 years increased between 2001-2005 and 2006-2010 from 42\% (36-48\%) to $58 \%(52-64 \%)$ and from $40 \%(35-46 \%)$ to $60 \%(55-65 \%)$, respectively. In contrast, the corresponding rates among patients aged $\geq 75$ years increased from only $25 \%(21-30 \%)$ to $33 \%(28-38 \%)$ and from $18 \%(14-21 \%)$ to $26 \%$ (22-30\%), respectively [2]. Similarly, in Sweden, 5-year RSRs (95\% CI) improved for patients aged 70-79 and $>79$ years from $24 \%(17-33 \%)$ to $75 \%(61-86 \%)$ and from $6 \%$ $(1-18 \%)$ to $25 \%(10-47 \%)$ between 1987-1993 and 2001-2008, respectively [10]. Of note, the RSRs in the Netherlands seems to lag compared with the Swedish rates. However, registration practices and the wideness of the CIs should be strongly considered when comparing survival rates across countries [12]. Therefore, a firm conclusion regarding potential survival disparities cannot be made. This topic provides an avenue for future research.

Congruent with our study, detailed data of the Swedish CML Registry among patients diagnosed during 2002-2010 showed that the application of TKIs increased over time among patients aged $\geq 70$ years [11]. Furthermore, during $2007-2009,85 \%$ and $79 \%$ of patients aged $70-79$ and $>79$ years received first-line TKI, respectively. Virtually all patients aged $\geq 70$ years diagnosed during 2014-2017 received first-line TKI, except for seven patients. The following reasons for not prescribing a TKI were reported: patients' preferences $(n=3)$, short lifeexpectancy $(n=1)$, functional status $(n=1)$, comorbidities $(n=1)$, and unknown reason $(n=1)$.

Despite these encouraging findings on survival improvement, elderly CML patients experience considerable excess mortality in contemporary clinical practiceparticularly those aged $\geq 80$ years. There are several possible contributing factors. Elderly patients may experience greater toxicities of TKI treatment due to comorbidities, which, in turn, might result in decreased adherence or discontinuation. Therefore, physicians were possibly reticent to offer TKIs to elderly, often comorbid patients-especially in the early years following commercially available imatinib. Whether the reticent application of TKI relates to the patients' comorbidity, frailty, desire, or other patient- or physician-related characteristics is an area for future research to further improve the care among the elderly. Improvement in CML management and supportive measures is desired, in the view of the rapid growth of the aging population, resulting in an increased incidence of CML among the elderly [13]. The current study provides a benchmark to assess whether excess mortality among elderly patients will decline in the years ahead.

The strength of our study includes the utilization of a nationwide population-based cohort with a relatively large number of patients, long-term study period, and comprehensive information on primary therapy. Therefore, unlike most population-based studies, we could directly link changing treatment practices with improvements in survival. Limitations of our study mainly pertain to the lack of detailed information on the response, monitoring, and subsequent treatment choices throughout most of the registry.

Collectively, in this nationwide, population-based study, RS increased significantly over time among various age groups of elderly CML patients. This improvement is likely accounted for by the introduction and increased application of TKIs since 2001. Nevertheless, elderly CML patients continue to experience excess mortality in a contemporary era with well-established TKI management. Forthcoming studies should center on further reducing excess mortality in this elderly population. 
Acknowledgements The authors would like to thank the registration clerks of the Netherlands Cancer Registry (NCR) for their dedicated data collection. The nationwide population-based NCR is maintained and hosted by the Netherlands Comprehensive Cancer Organisation (IKNL).

Author contributions AGD designed the study; GICGE analyzed the data; AGD supervised the data analyses; OV collected the data; GICGE wrote the paper with contributions from all authors, who also interpreted the data, and read, commented on, and approved the final version of the paper.

\section{Compliance with ethical standards}

Conflict of interest GICGE: no conflict of interest. OV: no conflict of interest. PEW: no conflict of interest. JJWMJ: Research Support Novartis, BMS. Honoraria Abbvie, Novartis, Pfizer, Incyte. NMAB: no conflict of interest. AGD: no conflict of interest.

Publisher's note Springer Nature remains neutral with regard to jurisdictional claims in published maps and institutional affiliations.

Open Access This article is licensed under a Creative Commons Attribution 4.0 International License, which permits use, sharing, adaptation, distribution and reproduction in any medium or format, as long as you give appropriate credit to the original author(s) and the source, provide a link to the Creative Commons license, and indicate if changes were made. The images or other third party material in this article are included in the article's Creative Commons license, unless indicated otherwise in a credit line to the material. If material is not included in the article's Creative Commons license and your intended use is not permitted by statutory regulation or exceeds the permitted use, you will need to obtain permission directly from the copyright holder. To view a copy of this license, visit http://creativecommons. org/licenses/by/4.0/.

\section{References}

1. Rohrbacher M, Berger U, Hochhaus A, Metzgeroth G, Adam K, Lahaye $\mathrm{T}$, et al. Clinical trials underestimate the age of chronic myeloid leukemia (CML) patients. Incidence and median age of $\mathrm{Ph} / \mathrm{BCR}-\mathrm{ABL}$-positive CML and other chronic myeloproliferative disorders in a representative area in Germany. Leukemia. 2008;23:602-4.

2. Pulte D, Redaniel MT, Bird J, Jeffreys M. Survival for patients with chronic leukemias in the US and Britain: age-related disparities and changes in the early 21 st century. Eur J Haematol. 2015;94:540-5.

3. Krok-Schoen JL, Fisher JL, Stephens JA, Mims A, Ayyappan S, Woyach JA, et al. Incidence and survival of hematological cancers among adults ages $\geq 75$ years. Cancer Med. 2018;7:3425-33.

4. Castagnetti F, Gugliotta G, Baccarani M, Breccia M, Specchia G, Levato L, et al. Differences among young adults, adults and elderly chronic myeloid leukemia patients. Ann Oncol. 2015;26:185-92.

5. Mandal R, Bolt DM, Shah BK. Disparities in chronic myeloid leukemia survival by age, gender, and ethnicity in pre- and postimatinib eras in the US. Acta Oncol. 2013;52:837-41.

6. Schouten LJ, Hoppener P, van den Brandt PA, Knottnerus JA, Jager JJ. Completeness of cancer registration in Limburg, The Netherlands. Int J Epidemiol. 1993;22:369-76.

7. Dickman PW, Adami HO. Interpreting trends in cancer patient survival. J Intern Med. 2006;260:103-17.

8. Ederer F, Heise H. Instructions to IBM 650 programmers in processing survival computations. Methodological Note No. 10. End Results Evaluation Section. National Cancer Institute: Bethesda, MD, 1959.

9. Dickman PW, Sloggett A, Hills M, Hakulinen T. Regression models for relative survival. Stat Med. 2004;23:51-64.

10. Bjorkholm M, Ohm L, Eloranta S, Derolf A, Hultcrantz M, Sjoberg J, et al. Success story of targeted therapy in chronic myeloid leukemia: a population-based study of patients diagnosed in Sweden from 1973 to 2008. J Clin Oncol. 2011;29:2514-20.

11. Hoglund M, Sandin F, Hellstrom K, Bjoreman M, Bjorkholm M, Brune $\mathrm{M}$, et al. Tyrosine kinase inhibitor usage, treatment outcome, and prognostic scores in CML: report from the populationbased Swedish CML registry. Blood. 2013;122:1284-92.

12. Sant M, Minicozzi P, Mounier M, Anderson LA, Brenner H, Holleczek B, et al. Survival for haematological malignancies in Europe between 1997 and 2008 by region and age: results of EUROCARE-5, a population-based study. Lancet Oncol. 2014;15:931-42.

13. Huang X, Cortes J, Kantarjian H. Estimations of the increasing prevalence and plateau prevalence of chronic myeloid leukemia in the era of tyrosine kinase inhibitor therapy. Cancer. 2012;118:3123-7. 